\title{
Assessing Locomotor Coordination during Walking and Running in Children and Adolescents: A Systematic Review
}

\author{
Goetschalckx $\mathbf{M}^{1 *}$, Moumdjian $\mathbf{L}^{1,2}$, Klingels $\mathbf{K}^{1}$, \\ Feys $P^{1}$ and Rameckers $E^{1,3}$ \\ ${ }^{1}$ REVAL Rehabilitation Research Center, Faculty of \\ Rehabilitation Sciences, Hasselt University, Hasselt, \\ Belgium \\ ${ }^{2}$ IPEM, Institute of Psychoacoustic and Electronic Music, \\ Faculty of Art and Philosophy, Gent University, Gent, \\ Belgium \\ ${ }^{3}$ CAPHRI, Maastricht University, Maastricht, the \\ Netherlands; Center of Expertise, Adelante Rehabilitation \\ Center, Valkenburg, the Netherlands \\ *Correspondling author: Goetschalckx M, Department \\ Rehabilitation Sciences and Physiotherapy, Hasselt \\ University, Agoralaan, Gebouw A, 3590 Diepenbeek, \\ Belgium
}

Received: February 08, 2021; Accepted: March 22, 2021; Published: March 29, 2021

\begin{abstract}
We aimed to systematically summarize assessment methods of locomotion coordination of the lower limbs in children, and to discuss the influence of person and task on locomotor coordination. Two databases (PubMed, Web of Science) were screened, up to April $1^{\text {th }} 2020$. Five articles were included. Locomotor coordination was assessed in Typically Developing Children (TD) and children with autism in different domains of coordination, using angle-angle plots, planar covariance, continuous relative phase and point-phasing. In TD children: age influenced intersegmental covariance when walking, and stability of temporal and amplitude phasing when running. Intersegmental covariance was influenced by vision and walking speed. Phase relationship was not influenced by weighted walking in autistic children, nor in TD children when walking backwards.
\end{abstract}

Keywords: Locomotion coordination; Child; Neurodevelopmental disorder; Walking; Running

\section{Abbreviations}

TD: Typically Developing Children; DCD: Developmental Coordination Disorder; ASD: Autism Spectrum Disorder; AXIS: Critical Appraisal Tool for Observational Cross-Sectional Studies; CCF: Cross-Correlation Function Analysis; CRP: Continuous Relative Phase; MARP: Mean Absolute Relative Phase; SDRP: Standard Deviation of the Relative Phase; DRP: Discrete Relative Phase

\section{Introduction}

Gait is a rhythmic cyclical movement that requires coordination between different body segments or joints to create an efficient and smooth movement pattern [1]. Coordination involves kinematic coupling, or synergy formation, in order to organize a movement pattern, to ensure stability under environmental demands [2,3]. Throughout literature, the term coordination is broadly used for closely linked constructs, e.g. movement control and skill. Movement control reflects the change in the scalar values of the function, for example the slow/faster scaling characteristics. Modifying coordination and control in order to adapt to environmental or task constraints, is rather an expression of skill. These constructs are however not independent from each other, and are therefore difficult to distinguish [4]. Krasovsky T et al. (2010) [1] suggested a specific definition for locomotor coordination: "An ability to maintain a context-dependent and phase-dependent cyclical relationship between different body segments or joints in both spatial and temporal domains." Within this definition of coordination, outcome measures can be divided into different subcategories [1]. Spatial and temporal domains can be distinguished, reflecting respectively the spatial relationship and temporal phasing relationship between different body segments or joints. Both spatial and temporal coordination can be measured according to an intrinsic reference frame, reflecting the coordination within the body (e.g. inter-joint coordination), or to an extrinsic reference frame, reflecting the coordination of body segments relative to the environment, describing positions of segments in external space (e.g. inter-limb coordination). We summarized the above definitions, based on [1], in a schematic overview which can be seen in Figure 1.

Typically, research in children focuses on the outcome of a gait task (e.g. performance score, time to complete a gait task), or traditional biomechanics (e.g. movements kinematics and kinetics of an individual joint or segment) to describe motor development and differences between population groups. When walking and running, differences between TD children and children with mild motor problems, for example children with Developmental Coordination Disorder (DCD), have been described, using traditional spatiotemporal parameters [5-10]. However traditional spatiotemporal gait parameters do not fit the above-mentioned definition of locomotor coordination. For example, walking faster would lead to a better time on task, but it does not necessarily imply a better coordination pattern. Besides, children with DCD fall more frequently than their TD peers, restricting participation in leisure activities [11]. In elderly, coordination impairments are associated with falling [12]. Integrating task outcome, biomechanics and locomotor coordination pattern can broaden our understanding of motor control mechanisms during gait [13], motor difficulties and frequent trips and falls in children with neurodevelopmental disorders, having mild motor problems. This systematic review will focus on the behavioral locomotion coordination patterns of walking and running and not on performance score of a gait task, traditional biomechanics, spatiotemporal gait parameters or isolated muscle activity patterns.
Phys Med Rehabil Int - Volume 8 Issue 2 - 2021

ISSN : 2471-0377 | www.austinpublishing group.com

Goetschalckx et al. () All rights are reserved
Citation: Goetschalckx M, Moumdjian L, Klingels K, Feys P and Rameckers E. Assessing Locomotor Coordination during Walking and Running in Children and Adolescents: A Systematic Review. Phys Med Rehabil Int. 2021; 8(2): 1177. 


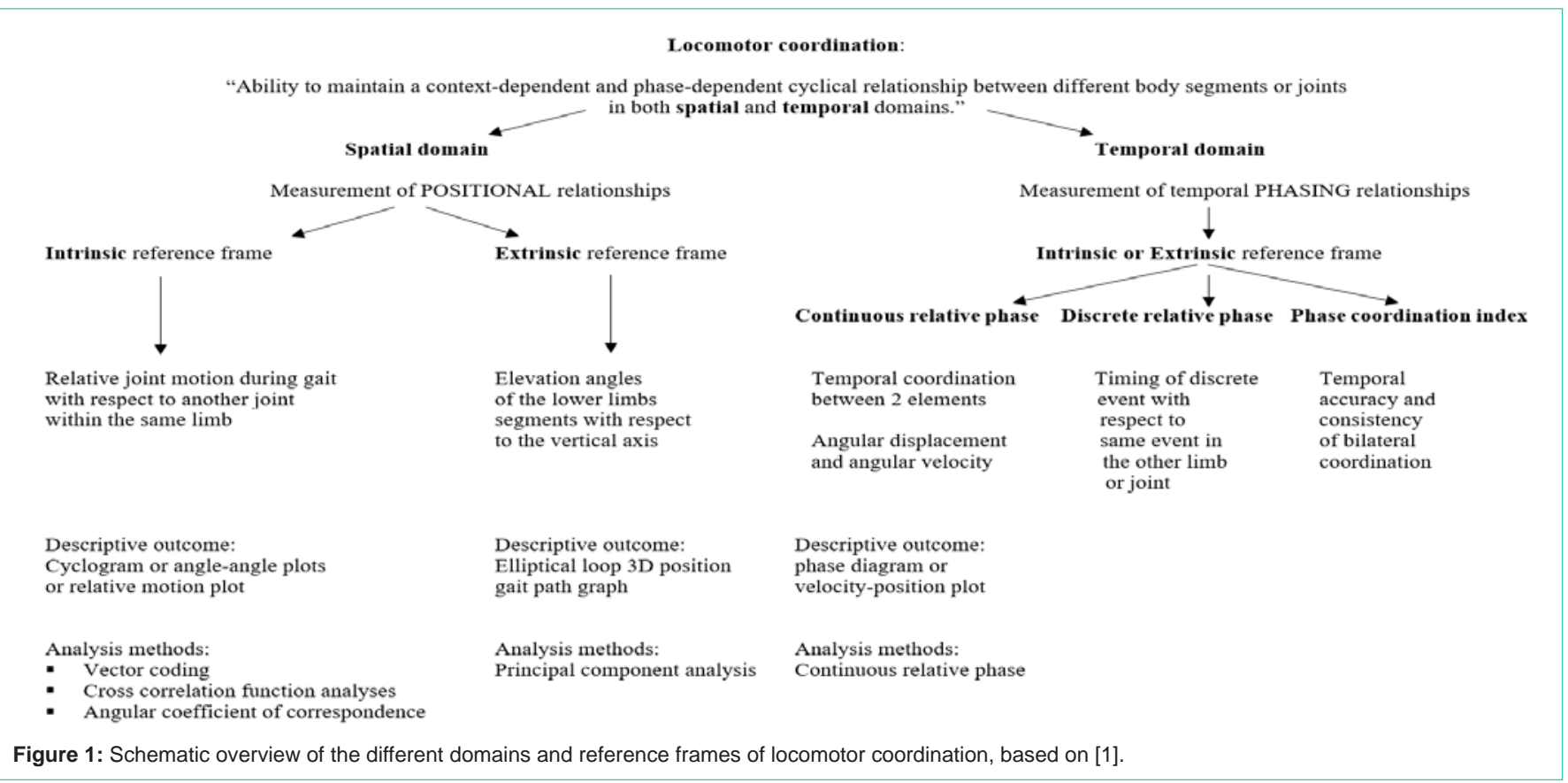

Previous reviews investigated locomotion coordination outcomes that are used in adults having motor disorders (e.g. neurological disorders and musculoskeletal disorders), and the influence of interventions targeting locomotor coordination $[14,15]$. These reviews reported that having a motor disorder (e.g. stroke, Parkinson disease, multiple sclerosis) has a detrimental role on locomotion coordination patterns and that interventions targeting locomotion coordination, could improve gait coordination. Similarly, investigating the locomotor coordination in children and adolescents with and without mild motor disorders (e.g. neurodevelopmental disorders) can give an insight in the typical and atypical characteristics of locomotor coordination patterns.

The inter- and intralimb coordination patterns are shaped by individual, task and environment conditions [16], which emphasizes the need to consider individual, task and environment conditions when interpreting coordination patterns. For example, walking in different environmental conditions (in dark or in well lighted) requires adaptability of coordination patterns to maintain stability and to achieve task outcome. However, this interaction between environment and task can also be affected by relevant person characteristics, such as age or having a motor impairment.

The current review will summarize locomotion coordination measures that were used in pediatric populations during walking and running, focusing on the outcomes that can be assigned into one of the domains and reference frames illustrated in the schematic overview (Figure 1). The review will focus on Typically Developing Children (TD) and children with neurodevelopmental disorders, without any neurological, musculoskeletal or cardiorespiratory disorder.

The aims of the systematic review are summarizing: a) the outcome measures that are used to assess locomotion coordination during walking and running; $b$ ) the person characteristics that are described (age, neurodevelopmental disorder) influencing locomotor coordination and c) the task characteristics, influencing locomotor

coordination.

\section{Methods}

\section{Search strategy}

The search was carried out in two electronic databases, PubMed and Web of Science, up to April $1^{\text {th }} 2020$. The search query compromised terminology related to "Coordination", "Gait" and "Children". The search strategy is provided in Appendix 1. The references of the included papers were checked for relevance. The systematic review has been registered in Prospero (CRD42020179748).

\section{Eligibility criteria}

Studies were included in the systematic review if they met the following criteria: a) outcome measure that assesses locomotor coordination pattern between different segments or joints of the lower limbs in spatial or temporal domain according the definition [1]; b) Study population were TD children or children with a neurodevelopmental disorder (DSM-V); c) The mean age of a study group was between 6-18 years; d) The coordination pattern was assessed during a continuous gait (walking or running) task, and e). The article full text was published in English.

Studies were excluded if: a) There was a lack of locomotor coordination pattern outcome between different segments or joints of the lower limbs, e.g. reporting only upper limb coordination patterns, only coordination patterns between upper limbs and lower limbs, or coordination patterns between pelvis or trunk, or coordination pattern of one joint or segment b) The coordination task was swimming, cycling or not continuous walking or running (e.g. obstacle crossing), c) The study assessed only muscle activation or coordination patterns using electromyography, d) The study population had a genetic syndrome, neurological, musculoskeletal, cardiorespiratory or endocrine disorder, e) The mean age of a study population group was below the age of six or above the age of 18 , f) The study only reported performance score of the gait task (e.g. 


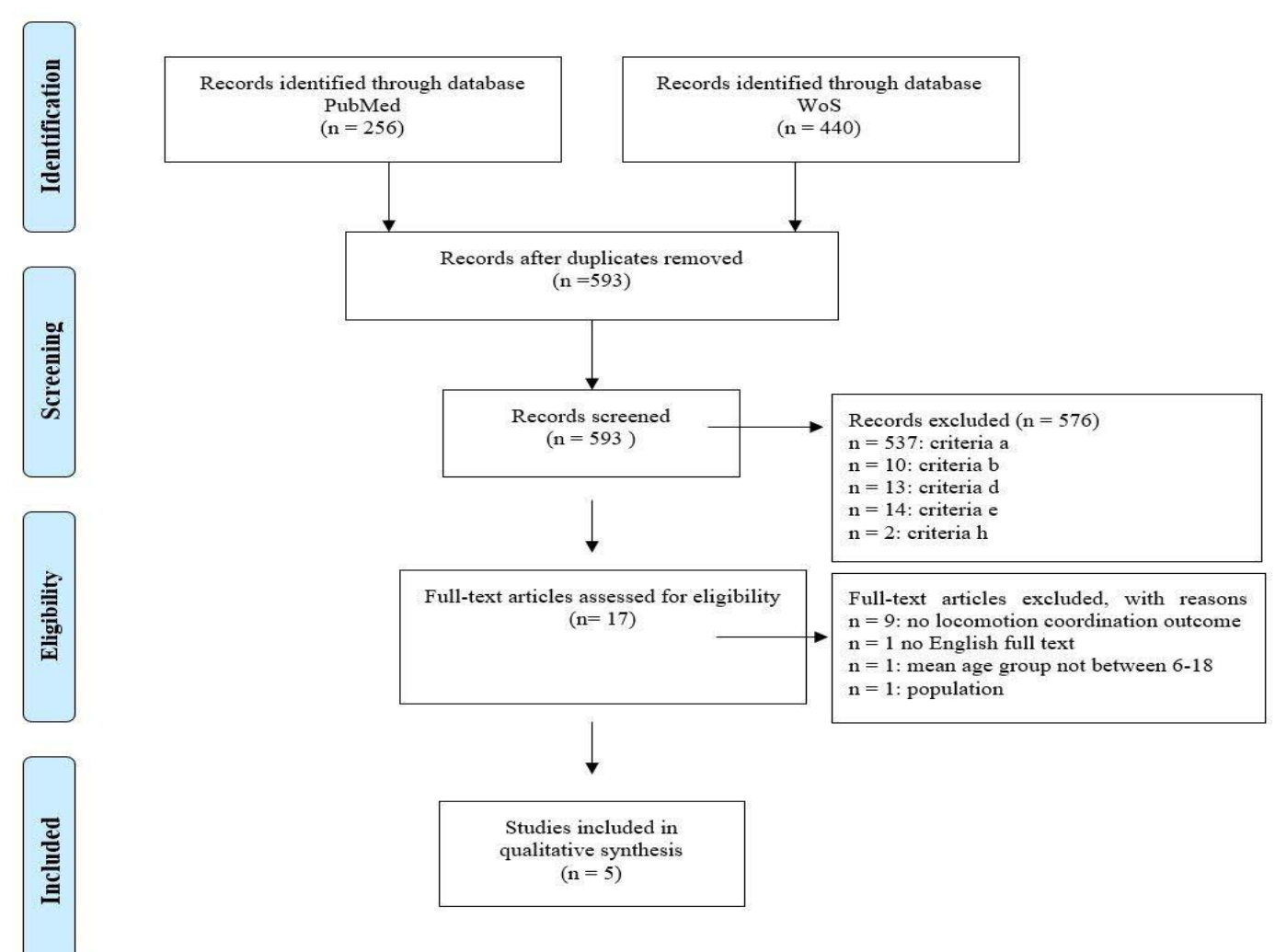

Figure 2: PRISMA flow diagram for study selection.

time to complete task, general motor test to assess coordination on ordinal scale); g) The study only reported individual kinematic or kinematics parameters of the gait task, h) The study was a conference proceedings, (systematic) review or technical paper, and if i) The study included animals.

Three independent researchers (MG, KH and SR) screened the title and abstracts of the identified articles for eligibility. A fourth reviewer (LM) was consulted in case of doubt for inclusion.

The following data were extracted from the selected studies: study population (typically developing, neurodevelopmental disorder), descriptive characteristics of the participants (age, gender) and number of participants. In addition, recording device, locomotor coordination outcome measures and person (age, neurodevelopmental disorder) and task characteristics (overground walking, treadmill walking, gait velocity, etc.) that were described, influencing locomotor coordination were extracted.

\section{Quality assessment}

To assess the quality of the included studies, the critical Appraisal Tool for observational Cross-Sectional Studies, AXIS, was used [17], focusing on five domains: Introduction, Methods, Results, Discussion and Other. For each criterion, a score of one was awarded when quality was present, establishing a total count of 20. Studies with a total score of $>15$ were considered as high quality, those between 1015 as moderate quality, and articles scoring $<10$ as poor quality [18]. Three independent researchers (MG, KH and SR) assessed the quality of the studies and results were compared afterwards.

\section{Results}

\section{Study selection}

The PRISMA flow diagram for study selection is presented in Figure 2. The search strategy yielded 256 hits in PubMed and 440 in Web of Science. After removal of duplicates, a total of 593 records were screened based on title and abstract. 576 articles were discarded based on inclusion criteria. The remaining 17 full text articles were screened for eligibility. However, thorough examination resulted in exclusion of 12 articles. Reference list of the remaining five articles were scanned for relevant literature. Finally, five articles were included in this systematic review.

\section{Quality assessment}

Results of the critical appraisal across the included studies are provided in Appendix 2. Overall, the quality of the studies was moderate (between 10-15 criteria are met). Concerning critical appraisal of items related to study population, only one study [19] reported sample size justification (item 3) and in three of the five studies, the study population structure did not represent the structure of the target population (e.g. only male or only female participants, more female compared to male participants) (item 5). Items about non-responders (item 7 and 14) were never mentioned.

\section{Study participant characteristics}

Five articles were included in this systematic review. Four studies included TD children [20-23]. One study included children with a neurodevelopmental disorder, namely Autism Spectrum Disorder (ASD) [19]. The age range was broad across the included 
Table 1: Overview of the study population, gait task (overground, treadmill), technical equipment and locomotor coordination outcome measure across the included studies.

\begin{tabular}{|c|c|c|c|c|c|c|c|c|c|c|}
\hline \multirow{3}{*}{ Article } & \multicolumn{3}{|c|}{ Study population } & \multirow[b]{3}{*}{ Overground } & \multirow[b]{3}{*}{ Treadmill } & \multirow{3}{*}{$\begin{array}{l}\text { Recording } \\
\text { device }\end{array}$} & \multicolumn{4}{|c|}{ Coordination outcome } \\
\hline & \multirow{2}{*}{$\begin{array}{c}\text { Neuro- } \\
\text { developmental } \\
\text { disorder } \\
\text { (n)/ Typical } \\
\text { developing (n) }\end{array}$} & \multirow{2}{*}{$\begin{array}{c}\text { Mean age } \pm \text { SD } \\
\text { (years) }\end{array}$} & \multirow{2}{*}{$\begin{array}{l}\text { Gender } \\
\text { (F\%) }\end{array}$} & & & & \multicolumn{2}{|c|}{ Spatial domain } & \multicolumn{2}{|c|}{ Temporal domain } \\
\hline & & & & & & & $\begin{array}{l}\text { Intrinsic reference } \\
\text { frame }\end{array}$ & $\begin{array}{l}\text { Extrinsic reference } \\
\text { frame }\end{array}$ & $\begin{array}{c}\text { Intrinsic } \\
\text { reference frame }\end{array}$ & $\begin{array}{c}\text { Extrinsic reference } \\
\text { frame }\end{array}$ \\
\hline $\begin{array}{c}\text { (Bleyenheuft \& } \\
\text { Detrembleur, } \\
\text { 2012) }\end{array}$ & TD (30) & $\begin{array}{lc}\text { 5 } & \text { age groups } \\
\bullet & 4.8 \pm 0.4 \\
\bullet & 9.3 \pm 0.5 \\
\bullet & 14.3 \pm 0.5 \\
-\quad & 23.5 \pm 2.9 \\
& 77.3 \pm 5.0\end{array}$ & $\begin{array}{l}\text { Mixed } \\
\text { (F: } \\
77 \%)\end{array}$ & & $\begin{array}{l}\text { Walking at } \\
3 \text { different } \\
\text { speeds } \\
\left(\mathrm{kmh}^{-1}\right): \\
1,3 \text { and } \\
5 \text { group } \\
5 \text {-years } \\
\text { old: unable } \\
\text { to walk } \\
5 \mathrm{kmh}^{-1}\end{array}$ & $\begin{array}{l}\text { ELITE system, } \\
6 \text { infrared } \\
\text { cameras } \\
\text { placed around } \\
\text { the treadmill, } \\
\text { recording } \\
\text { coordinates } \\
\text { every } 10 \mathrm{~ms} \\
\text { in 3D }\end{array}$ & na & $\begin{array}{l}\text { Elevation angles } \\
\text { relative to the vertical } \\
\text { - Thigh: } \\
\text { - Shank } \\
\text { Planar covariation } \\
\text { (Borghese et al. 1996). } \\
\text { PCA: PC1, PC2, PC3 } \\
\text { \%variance PC1, PC2 } \\
\text { and PC3 }\end{array}$ & na & na \\
\hline $\begin{array}{c}\text { (Hallemans \& } \\
\text { Aerts, 2009) }\end{array}$ & TD (60) & $\begin{array}{lc}3 \text { 3 } & \text { age groups } \\
- & 5.14 \pm 0.98 \\
- & 9.12 \pm 1.23 \\
& 28.00 \pm 7.00\end{array}$ & $\begin{array}{l}\text { Mixed } \\
\text { (F: } \\
50 \%)\end{array}$ & $\begin{array}{l}\text { Walking barefoot over } \\
\text { instrumented walkway } \\
(1.5 \times 11 \mathrm{~m}) \text { at self- } \\
\text { selected speed. } \\
2 \text { conditions: } \\
-\quad \text { full vision } \\
5 \text { walking trials as } \\
\text { habituation next } 3 \text { trials } \\
\text { are used for analysis }\end{array}$ & & $\begin{array}{l}\text { Vicon Mcam } \\
60,6 \text { cameras, } \\
120 \mathrm{~Hz} \text {, Vicon } \\
\text { Motion system }\end{array}$ & $\begin{array}{l}\text { Angle-angle plots } \\
\text { - Shank-thigh } \\
\text { Perimeter, area, } \\
\text { shape, orientation } \\
\text { and distance of the } \\
\text { centroid of the loop to } \\
\text { the origin. } \\
\text { Inter-trial variability in } \\
\text { shape and orientation } \\
\text { of the angle-angle } \\
\text { plots. } \\
\text { Cross correlation } \\
\text { function analysis } \\
\text { (CCF). }\end{array}$ & $\begin{array}{l}\text { Elevation angles } \\
\text { relative to the vertical } \\
\text { and heading direction: } \\
\text { - Thigh } \\
\text { - Shank } \\
\text { Planar coveratiation } \\
\text { (Borghese et al. 1996). } \\
\text { PCA: PC1, PC2, PC3 } \\
\text { Variability of PC1, } \\
\text { PC2, PC3 } \\
\text { Eigenvectors u1, } \\
\text { u2, u3 } \\
\text { - planarity index } \\
\quad \text { shape of gait } \\
\text { - loop } \\
\text { Cross Correlation } \\
\text { Function (CCF) } \\
\text { analysis }\end{array}$ & na & na \\
\hline $\begin{array}{l}\text { (Meyns et al., } \\
\text { 2013) }\end{array}$ & TD (28) & $\begin{array}{l}2 \text { age groups } \\
-\quad 9.4 \pm 2.16 \\
-\quad 29.86 \pm 6.22\end{array}$ & $\begin{array}{l}\text { Mixed } \\
\text { (F: } \\
50 \%)\end{array}$ & $\begin{array}{l}\text { Walking along a } 10 \mathrm{~m} \\
\text { walkway at preferred } \\
\text { speed } \\
2 \text { conditions: } \\
\text { - forward } \\
3 \text { successful walking } \\
\text { trials for each condition }\end{array}$ & & $\begin{array}{l}\text { Vicon motion } \\
\text { system, } 8 \\
\text { cameras, } \\
100 \mathrm{~Hz}, \\
\text { Total body } \\
\text { "PluglnGait" } \\
\text { marker set }\end{array}$ & na & na & na & 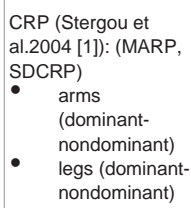 \\
\hline (Whitall, 1989) & TD (40) & $\begin{array}{ll}5 \text { age groups } \\
\bullet & 2.8 \pm 0.4 \\
\bullet & 4.2 \pm 0.3 \\
\bullet & 6.4 \pm 0.3 \\
\bullet & 9.4 \pm 0.3 \\
- & 26.1 \pm 4.8\end{array}$ & $\begin{array}{l}\text { Only F } \\
(100 \%)\end{array}$ & $\begin{array}{l}\text { Running at preferred } \\
\text { speed, outside, at level } \\
\text { ground with no weight } \\
\text { perturbation practice } \\
\text { trial } 2 \text { trials filmed for } \\
\text { analysis (4-8 strides) }\end{array}$ & & \begin{tabular}{|l}
$16 \mathrm{~mm}$ \\
Photosonics \\
intermittent \\
pin-registered \\
camera, $64 \mathrm{fps}$, \\
$16 \mathrm{~mm}$ focal \\
length lens, \\
positioned \\
perpendicular \\
to the runway, \\
filming from a \\
sagittal view
\end{tabular} & na & na & na & $\begin{array}{l}\text { Temporal and } \\
\text { amplitude phasing } \\
\text { measure between } \\
\text { limbs (point phase) } \\
\text { - } \quad \text { step time/stride } \\
\text { time } \\
\text { - } \quad \text { step length/ } \\
\text { stride length } \\
\text { mean and variability } \\
\text { phasing }\end{array}$ \\
\hline
\end{tabular}

Abbreviations: TD: Typically Developing; ASD: Autism Spectrum Disorder; F: Female, YO: Years Old; PCA: Principal Component Analysis; CRP: Continuous Relative Phase; MARP: Mean Absolute Relative Phase; SDRP: Standard Deviation of Relative Phase; CCF: Cross Correlation Function Analysis; BP: Backpack; WV: Weight Vest; FPS: Frames per Second; NA: Not Applicable.

studies, including children with an age of 2 up to adults and elderly. Subgroups of participants younger than age 6 and older than age 18, were included in four studies [20-23]. However, in this systematic review, only results concerning subgroups with mean age from 6 up to 18 are reported.

\section{Task characteristics}

Four studies investigated locomotor coordination during walking, whereas one examined locomotor coordination during running overground at preferred speed [23]. Treadmill walking at 3 different speeds $\left(1,3\right.$ and $\left.5 \mathrm{kmh}^{-1}\right)$ was performed in one study [22]. The other three studies examining walking, used overground walking along an instrumented walking of approximately 10 meters and participants were instructed to walk at preferred self-selected speed [19-21].

Each of the included studies using walking, investigated the influence of a task condition on locomotor coordination pattern: walking at three different speeds [22], or walking with and without 
vision [21], or forward and backward walking [20], or weighted walking [19]. The influence of these different task conditions on the investigated locomotor coordination outcome will be described later.

\section{Measures of locomotion coordination}

Two articles assessed locomotion coordination during walking in the spatial domain [21,22]. Hallemans et al. (2009) [21] assessed coordination in spatial domain according to an intrinsic reference frame. An infrared retroflective 6-camera system (Vicon Motion system) recorded segmental kinematics during overground walking. The outcome measure was the coupling between the thigh and shank, and the shank and foot segments. By plotting the motion of the shank relative to the thigh motion, and the foot relative to the shank motion, angle-angle plots were formed to investigate the coupling between the thigh and shank, and the shank and foot segments [21]. Descriptive characteristics of the angle-angle plots were determined, namely, perimeter, area, shape, distance to centroid of the loop to origin, orientation and inter-trial variability of the angle-angle plots. Besides, Cross-Correlation Function Analysis (CCF) was used to examine peak CCF and time lags, representing the match between the plots. The spatial domain of locomotor coordination according to an extrinsic reference frame was assessed by Bleyenheuft et al. (2012) and Hallemans et al. (2009) [21,22]. Infrared camera system (ELITE system) was used by Bleyenheuft et al. (2012) [22] to record the segmental kinematics in the sagittal plane during treadmill walking and an infrared retroflective 6-camera system (Vicon Motion system) was used by Hallemans et al. (2009) [21] during overground walking. The outcome measure of interest were the elevation angles of the lower limbs, representing the absolute orientation of the segment with respect to the vertical axis and the heading or walking direction. When plotting the elevation angles against each other in $3 \mathrm{D}$ position space, the obtained elliptical loop represents the kinematic segmental covariation between the lower limb segments. The degree of interdependency or coupling between the lower limb segments was further analyzed by planar covariation technique, according to Borghese et al. (1996) [24]. The planarity index, a measure of planarity, is one of the co-variation plane parameters, that is equal to the cumulative variance of the variability of the first and second principal component $(\mathrm{V} 1+\mathrm{V} 2)$. Besides, the covariation plane orientation was calculated (cosine angle between the plane normal and the thigh axis), reflecting the contribution of the thigh angle to the plane orientation. Lastly, the shape of the gait loop, representing the degree of correlation and coupling between the thighs, shank and foot elevation angles (characterized by the ratio of V1 and V2), was calculated.

Temporal domain of coordination was measured in three studies $[19,20,23]$. Eggleston et al. (2018) [19] assessed temporal domain of coordination according to an intrinsic reference frame. A 10-camera Motion capture system (Vicon Motion system) obtained 3D segmental kinematics during overground walking. The outcome measure was coordination between thigh-leg, leg-foot and thigh-foot segments, by examining the difference in phase planes between the lower limb segments within one limb. First, segmental angular displacement and angular velocity are normalized to account for different amplitude and frequency between signals $[25,26]$. Afterwards phase angles could be calculated from the normalized angular positions and velocities. Phase angles are further used to analyze the Continuous
Relative Phase (CRP) between two segments, describing the temporal coordination between thigh-leg, leg-foot and thigh-foot throughout the data series [19]. The temporal domain of coordination was assessed accordingly to an extrinsic reference frame by Meyns et al. (2013) [20] and Whitall et al. (1989) [23]. Meyns et al. (2013) [20] used 8-camera motion capture system (Vicon motion system) to track 3D full-body kinematic data. The authors assessed the temporal coordination between both legs by calculating the CRP between the lower limbs [20]. The mean relative phase over the gait cycle, indicating the timing of the interlimb movements, was expressed by the Mean Absolute Relative Phase (MARP). Also, the coordinative stability, expressed as the Standard Deviation of the Relative Phase (SDRP), was calculated. Lastly, Whitall et al. (1989) [23] used a $16 \mathrm{~mm}$ Photosonics intermittent pin-registered camera to film the subjects at 64 frames per second from a sagittal view. The temporal and amplitude phasing between the lower limbs were calculated, using a point phase technique, resembling more to the discrete relative phase, where after the mean and variability of the phasing was determined (Table 1 and 2) [23].

Four articles examined the influence of age on locomotor coordination patterns of the lower limbs while walking [20-23]. When comparing spatial coordination according to intrinsic reference frame between different age group (3-6 years old, 7-11 years old, adults), Hallemans et al. (2009) [21] used angle-angle plots to investigate the coupling between the thigh and shank, and the shank and foot segments while walking overground at self-selected speed. The authors reported a significant larger distance of centroid to origin of the shank-foot plot in adults compared to both 3 to 6 year old and 7 to 11 year old children, which can be the result from a difference in mean elevation angle of the shank or foot segment, or both segments. When comparing spatial coordination according to extrinsic reference frame between different age groups, the study of Hallemans et al. (2009) [21] found that the plane covariation orientation was significantly smaller in the 3 to 6 years old children compared to the 7 to 11 years old children, indicating that the thigh angle contributes less to the orientation of the plane in younger children. Besides a negative relationship between plane covariation orientation and dimensionless walking speed existed, suggesting that the thigh angle is a more important contributor to the plane covariation orientation when walking slower. Both Hallemans et al. (2009) and Bleyenheuft et al. (2012), found no significant differences between age groups in global form or shape of the gait loop and planarity index when walking at self-selected speed [21] or when walking at lowest imposed treadmill speed of $1 \mathrm{kmh}^{-1}$ [22]. The study of Bleyenheuft et al. (2012) imposed different treadmill speeds $\left(1 \mathrm{kmh}^{-1}, 3 \mathrm{kmh}^{-1}\right.$ and $\left.5 \mathrm{kmh}^{-1}\right)$ [22]. At the highest treadmill speed $\left(3 \mathrm{kmh}^{-1}\right)$ achieved by the 5 years old children the shape of the gait loop did differ significantly between the 5 years old and the 10 years old children. When increasing treadmill speed up to $5 \mathrm{kmh}^{-1}$, the planarity of the loop did significantly differ between 10 years old children and the older age groups, indicating that the segmental covariation of the lower limbs while walking might be acquired soon at spontaneous speed, but later at higher imposed treadmill speeds.

When comparing temporal coordination according to extrinsic reference frame between different age groups, Meyns et al. (2013) observed no significant differences between MARP and the SDRP in 
Table 2: Overview of the person and task characteristics investigated in the included studies and the influence of these characteristics on locomotion coordination pattern.

\begin{tabular}{|c|c|c|c|c|c|c|}
\hline \multirow{2}{*}{ Article } & \multicolumn{2}{|c|}{ Person characteristics } & \multicolumn{4}{|c|}{ Task characteristics } \\
\hline & $\begin{array}{l}\text { Neurodevelopmental } \\
\text { disorder }\end{array}$ & Age & Gait velocity & Vision & Weighted walking & Walking direction \\
\hline $\begin{array}{l}\text { (Bleyenheuft \& } \\
\text { Detrembleur, } \\
\text { 2012) }\end{array}$ & na & $\begin{array}{l}\text { Age groups: } 5,10,15,20,70 \text { yo } \\
\text { PV1 and PV2 no significant } \\
\text { difference between age group } \\
\text { of } 10 \text { and older groups at all } \\
\text { treadmill speeds. } \\
\text { PV3 significant difference } \\
\text { between age group of } 10 \text { and } \\
15 \text { years, and } 10 \text { and } 20 \text { years } \\
\text { at higher speeds }\left(5 \mathrm{kmh}^{-1}\right) \text {. }\end{array}$ & $\begin{array}{l}\text { Treadmill walking velocity: } 1 \text {, } \\
\text { 3, } 5 \mathrm{kmh}^{-1} \\
\text { At } 1 \mathrm{kmh}^{-1} \text { no significant } \\
\text { differences in PV1, } \\
\text { PV2 or PV3 between } \\
\text { groups. } \\
\text { At higher speed ( } 3 \mathrm{kmh}^{-} \\
\text {1) significant differences } \\
\text { in PV1 and PV2 } \\
\text { between age groups of } \\
5 \text { and } 10 \text { years. } \\
\text { At the highest walking } \\
\text { speed ( } 5 \mathrm{kmh}^{-1} \text { ), } \\
\text { significant difference in } \\
\text { PV3 between age group } \\
\text { of } 10 \text { and } 15 \text { years, and } \\
10 \text { and } 20 \text { years. }\end{array}$ & na & na & na \\
\hline $\begin{array}{c}\text { (Hallemans \& } \\
\text { Aerts, 2009) }\end{array}$ & na & $\begin{array}{l}\text { Age groups: } 3-6 \text { yo, } 7-11 \text { yo, adults } \\
\text { Planar covariation: } \\
\text { Plane covariation orientation } \\
\text { smaller in 3-6yo compared } \\
\text { to 7-11yo. } \\
\text { Angle-angle plots: } \\
\text { Distance centroid shank- } \\
\text { foot is smaller in children } \\
\text { compared to adults. }\end{array}$ & na & 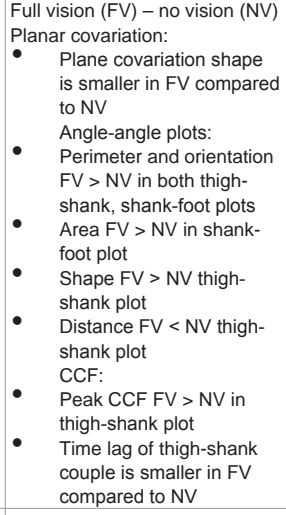 & na & na \\
\hline $\begin{array}{l}\text { (Meyns et al., } \\
\text { 2013) }\end{array}$ & na & $\begin{array}{l}\text { Age: children }(9.40 \text { yo), adults } \\
\text { CRP: MARP, SDRP } \\
\text { No significant effect on MARP } \\
\text { or coordination stability } \\
\text { (SDCRP). }\end{array}$ & na & na & na & $\begin{array}{l}\text { Forward (FW) - backward } \\
\text { (BW) } \\
\text { CRP: MARP, SDRP } \\
\text { No significant } \\
\text { difference } \\
\text { in interlimb } \\
\text { coordination MARP } \\
\text { and SDCRP }\end{array}$ \\
\hline $\begin{array}{l}\text { (Eggleston et } \\
\text { al., 2018) }\end{array}$ & $\begin{array}{l}\text { autism spectrum disorder } \\
\text { no control group }\end{array}$ & na & na & na & $\begin{array}{l}\text { No weight (NW), backpack } \\
\text { (BP), weighted vest (WV) } \\
\text { Group analysis; } \\
\text { No large ES among } \\
\text { any of the condition } \\
\text { comparisons } \\
\text { Single-subject analysis: } \\
\text { Small ES among the } \\
\text { condition. comparisons: } \\
\text { largest number of large } \\
\text { ES was for NW-BP } \\
\text { comparison. }\end{array}$ & na \\
\hline (Whitall, 1989) & na & $\begin{array}{l}\text { 8 age groups: } 2.5-3.5 ; 3.5-4.5 ; 6-7 ; \\
\text { 9-10; adults (26.1 } 4.8) \\
\text { Mean phasing and variability } \\
\text { of phasing: main effect of age } \\
\text { Temporal and amplitude } \\
\text { phasing of the run is situated } \\
\text { around } 50 \% \text {. } \\
\text { No effect for age for mean } \\
\text { temporal phasing. } \\
\text { Variability of temporal phasing } \\
\text { decreased with age increase: } \\
3 \text { and } 4 \text { yo > adults (difference } \\
<1.5 \%) \text {. } \\
\text { Univariate effect for age is } \\
\text { significant for mean amplitude } \\
\text { phasing ( } 9 \text { and } 18 y 0>\text { other } \\
\text { age groups). } \\
\text { Variability of amplitude } \\
\text { phasing decreased across } \\
\text { age. }\end{array}$ & na & na & na & na \\
\hline
\end{tabular}

Abbreviations: F: Female; YO: Years Old; PCA: Principal Component Analysis; CRP: Continuous Relative Phase; MARP: Mean Absolute Relative Phase; SDRP: Standard Deviation of Relative Phase; CCF: Cross Correlation Function Analysis; NA: Not Applicable. 
children and adults, when walking [20]. Also, the study of Whitall et al. (1989), using point phase technique, did not found a main effect of age on mean temporal and amplitude phasing during running. However, the variability of temporal and amplitude phasing when running decreased across age [23].

The influence of walking with or without vision was investigated by Hallemans et al. (2009) [21]. Participants walked barefoot over an instrumented walkway, free from obstacles, at self-selected speed in full vision and no vision condition. Swimming glasses were masked with black tape to occlude vision during the no vision condition. A significant effect was found for intersegmental coordination, using the planar covariation technique, between conditions. Namely, the shape of the gait loop was smaller in the full vision condition than in the no vision condition. When investigating separately the coupling between thigh-shank, and shank-foot segments, using angleangle plots, the perimeter of both the thigh-shank, and shank-foot segments were smaller in the no vision condition compared to the full vision condition. Besides, the perimeter was positive related to dimensionless walking speed. Only in the thigh-shank couple, the shape and orientation of the angle-angle plot were smaller in the no vision condition, while the distance of the centroid to origin is significantly larger. The orientation of the thigh-shank plot was negatively related to dimensionless walking speed. In the shankfoot couple, the area of the angle-angle plot was smaller during the no vision condition, which was positively related to dimensionless walking speed. Besides, visual condition had a significant effect on the cross correlation between thigh-shank. In the no vision condition, the cross correlation was significant smaller and the time lag was significantly larger. The cross correlation between the shank-foot was not affected by vision, but a positive relationship was observed between walking speed and cross correlation of the shank-foot.

Walking direction was examined in the study of Meyns et al. (2013) [20]. Participants were instructed to walk at their preferred speed along a 10 meter walkway either forward or backward. Walking backward had no significant effect on mean relative phase and coordinative stability.

Lastly, the effect of weighted walking in children with ASD on intralimb coordination, using CRP between thigh-leg, leg-foot and thigh-foot segments, was examined by Eggleston et al. (2018) [19]. Children walked overground on a straight-line distance of approximately nine meters, in three conditions: no added mass, while wearing a modified weight vest with $15 \%$ of their body weight, and while wearing a backpack loaded with $15 \%$ of their body weight. Generally, there were few significant differences between the CRP, but no large effect sizes were found in CRP between the unloaded versus loaded conditions. When looking at single-subject analysis, a large percentage of significant difference were found among individual participants, which were more presented in the unloaded condition compared to the loaded conditions. However, also in the single-subject analysis, only small effect sizes found, leading to no meaningful differences between conditions.

\section{Discussion}

The aim of the study was to systematically review the existing assessments to examine the locomotor coordination of the lower limbs during walking and running in typically developing children and adolescents and with neurodevelopmental disorder during gait tasks and to understand the influence of person and task characteristics on locomotor coordination patterns. Locomotor coordination was based on the definition of Krasovsky et al. (2010) [1]. When screening articles, most of the records were excluded because there was a lack of locomotor coordination pattern outcome between different segments or joints of the lower limbs, confirming that the term coordination is broadly used for closely linked constructs, e.g. movement control and skill [4].

In this review five articles assessing locomotor coordination of the lower limbs during a gait task were included. Although four studies included participants younger than age 6 or older than age 18 , only results concerning the group analysis aged between 6 and 18 are reported in the review. Therefore, results of 66 TD children, aged between 6-15 years, and results of ten children with autism, mean age of 11 years, are included. Three of the five included articles used relative phase (continuous relative phase or discrete relative phase), to assess the temporal domain of locomotor coordination during gait tasks in children. The other two articles assessed the spatial domain of locomotor coordination during gait tasks, using planar covariation technique. Depending on the aim, examining the spatial relationship or more the temporal relationship between different body segments or joint, a different locomotor coordination outcome measure can be used. For example, if the goal is to provide a more qualitative description of a relative joint motion with respect to another joint in the same limb, angle-angle plots can be used. When the consistency of spatial locomotor coordination is more of interest, the angular coefficient of correspondence can be computed. If the aim is to assess the temporal phase relationship between two joints across the gait cycle, CRP needs to be calculated. A disadvantage of CRP is the need for normalization, which can be performed using different techniques [27]. In contrast to CRP, DRP requires no normalization. The DRP assess the timing of a discrete event with respect to the same event in another limb or joint, and therefore yield one value per gait cycle, and might be less sensitive than CRP which quantifies continuous relationships between different segments or joints. One outcome measure, namely Phase Coordination Index (PCI), has not yet been applied in children. The PCI encompass both accuracy and consistency of bilateral temporal coordination [28]. The PCI can, in contrary to the other locomotor coordination outcomes, be assessed using more clinically available recording devices (e.g. wearable sensors) [29]. The schematic overview of the different domains of locomotor coordination can guide researchers and clinicians in defining the corresponding outcome measure. The different outcome measures described in Figure 1 cannot completely describe the construct of locomotor coordination. Measuring both spatial and temporal domain of locomotor coordination will provide the most comprehensive assessment of the construct of locomotor coordination because a change in temporal phase coordination does not necessitate a change in the temporal amplitude coordination. In addition, little research concerning psychometric properties of these outcomes is available in children $[1,14]$. Therefore, caution is needed when interpreting the results.

It is required to consider specific task, person and environmental characteristics when interpreting the coordination pattern. Based on the described task and person characteristics in the included 
articles, it seems that gait speed, visual information and age are factors influencing locomotor coordination pattern in TD children. Therefore, assessing locomotor coordination in different task conditions, gives knowledge of the ability to adapt the coordination pattern and movement control scalar values to accomplish the task. In the following paragraph, the influence of different task characteristics and age on locomotor coordination pattern is discussed.

The influence of visual information on locomotor coordination pattern was assessed by Hallemans et al. (2009) [21]. The absence of visual information while walking overground reduced the coupling between thigh-shank and resulted in a more out-of-phase control [21]. The changes in phasing relationship between segments has been found to affect postural stability [30]. The fact that shape of the gait loop and phasing relationship are more affected by visual deprivation than the strength of the coupling, suggests that active neural control plays a role in planar covariation of thigh-shank and that the differences in phasing relationship might reflect adaptations in postural control strategies $[31,32]$. In contrast to the coupling between thigh-shank, the coupling between foot-shank segments seems primarily dependent on walking speed, and not vision. The increase in CCF implies that an increase in walking speed resulted in a stronger coupling between shank-foot segments, suggesting that the shank and foot coupling is more reinforced by mechanical factors, than the absence or presence of visual information [30].

Moreover, gait velocity seems to have an influence on intralimb coordination assessed by planar covariance. At spontaneous walking speed of $1 \mathrm{kmh}^{-1}$, the gait loop and planarity of the gait loop are adult like in children even at the age of 5 years old, but later when a higher walking speed is imposed by a treadmill [22]. Planar covariance during walking at self-selected speed was investigated by Hallemans et al. (2009), and reported no difference across age groups in planarity index [21]. Also Cheron et al. (2001), examined planar covariance in children (11 months to 144 months old) and adults, and observed that the planarity approaches its mature pattern faster than does the shape of the gait loop when walking at comfortable tempo [33]. However, Hallemans et al. (2009) [21] observed that the orientation of the gait loop differed between age groups, indicating a lower contribution of the thigh to the orientation of the plane in the younger children ( 3 to 6 years old). These results suggest a relationship between age and planar covariance, influenced by walking speed, which highlights the need to integrate walking speed when interpreting results of locomotor coordination.

The influence of walking direction on temporal interlimb coordination was investigated by Meyns et al. (2013) [20]. Walking backward had no significant effect on the mean and stability of temporal interlimb coordination, investigated by mean CRP and standard deviation of CRP. Special attention was paid during backwards walking to minimize head turns, minimizing visual information of the walking path. Together with the results of Hallemans et al. (2009) [21], it seems that exclusion of visual feedback has an impact on the amplitude and a change in spatial phasing relationship between thighshank couple, but not on temporal interlimb coordination stability of the lower legs $[20,21]$.

Only one study included children with autism spectrum disorder [19], however without including a comparison group of TD children.
Results suggested that weighted walking, did not influence lower extremity intersegmental coordination in children with autism. Although walking with weight vest is commonly used by therapists using sensory-integration therapy. Further research is warranted to investigate its clinical application for locomotor coordination problems during walking $[19,34]$.

Lastly, temporal locomotor coordination pattern of the lower limbs during running was investigated by only one study [23]. Using a point-phasing technique, a decrease across age in variability of temporal and amplitude phasing when running was reported. Special attention is needed when interpreting these results [23]. Participants were filmed by a $16 \mathrm{~mm}$ Photosonics intermittent pin-registered camera, which could be less reliable and valid than the new 3D motion capture systems used in the other included studies [35]. However, these laboratory-based measurement instruments are less useful in clinical settings. Therefore, further research is recommended to investigate the use and accuracy of recording devices that are available for clinicians (e.g. accelerometers, foot switches and wearable sensors) for measuring locomotor coordination.

Despite only one study investigated locomotor coordination during running, many sports and games consist of running, a complex movement pattern characterized by sequencing of the lower limb segments to propel the body forward and to maintain balance [36]. Studies investigating running biomechanics and leg muscle activation in children with coordination deficits, observed that these children have a greater variability in sagittal kinematics of the lower limbs, deficit ankle power generation and different leg muscle activation patterns than TD children $[9,10,37]$. These results suggest that children with coordination deficits have difficulties in controlling their lower limbs to generate an efficient running strategy. Investigating locomotor coordination pattern during running can give more information of the motor control mechanisms during gait [13].

Critical comments and quality of the individual studies were appraised by the AXIS tool. In general, the quality of the studies was moderate (between 10-15 criteria are met). Even so, in three studies, the study population did not represent the structure of the target population concerning sex differences. (e.g. only male or only female participants, more female compared to male participants) (Item 5). Nevertheless, there is no clear evidence that sex has an influence on locomotor coordination. The small sample size of the group analysis reduced power in most of the studies.

The systematic review has limitations. Only five articles met the specific inclusion criteria. The limited number of studies, insufficient power and moderate quality studies are limitations in terms of conclusions that can be made regarding the locomotion coordination outcomes during walking and running in children and adolescents. Furthermore, limited evidence concerning psychometric properties of these outcomes is available in pediatric populations, subsequently, results have to be interpreted with caution.

Further high quality studies examining psychometric properties of locomotor coordination outcomes and accuracy of measuring devices when walking and running in both TD children and children with neurodevelopmental disorders, is recommended. Information about locomotion coordination patterns can help clinicians and 
researchers interpreting walking difficulties and falls in children with neurodevelopmental disorders and guiding therapy targeting locomotion coordination.

\section{Conclusion}

The systematic review summarized the locomotion coordination measures that were used in pediatric population, focusing on the outcomes that could be assigned more into spatial or temporal domains of the construct of locomotor coordination. Before assessing locomotor coordination, it is imperative to define the specific aim and domain of interest of locomotor coordination. However, the most comprehensive assessment of the construct locomotor coordination includes the measurement of both spatial and temporal domain of locomotor coordination. Specific task characteristics and age is required when interpreting the coordination pattern.

\section{Acknowledgements}

We would like to acknowledge master thesis students (KH, SR) rehabilitation science and physiotherapy from Hasselt University for their assistance in screening articles and quality assessment.

\section{Funding}

This research did not receive any specific grant from funding agencies in the public, commercial, or not-for-profit sectors.

\section{References}

1. Krasovsky T, Levin MF. Review: toward a better understanding of coordination in healthy and poststroke gait. Neurorehabil Neural Repair. 2010; 24: $213-$ 224.

2. Kelso JA. Phase transitions and critical behavior in human bimanua coordination. The American journal of physiology. 1984; 246: R1000-1004.

3. Bernstein N. The Co-ordination and Regulation of Movements: Oxford: Pergamon Press. 1967

4. Newell KM. Coordination, Control and Skill. In: D. Goodman RBW, IM Franks editor. Differing Perspectives in Motor Learning, Memory, and Control. NorthHolland: Elsevier Science Publishers BV. 1985: 295-317.

5. Deconinck FJ, De Clercq D, Savelsbergh GJ, Van Coster R, Oostra A, Dewitte $\mathrm{G}$, et al. Differences in gait between children with and without developmental coordination disorder. Motor Control. 2006; 10: 125-142.

6. Wilmut K, Du W, Barnett AL. Gait patterns in children with Developmental Coordination Disorder. Experimental brain research. 2016; 234: 1747-1755.

7. Du W, Wilmut K, Barnett AL. Level walking in adults with and withou Developmental Coordination Disorder: An analysis of movement variability. Hum Mov Sci. 2015; 43: 9-14.

8. Chia LC, Licari MK, Guelfi KJ, Reid SL. Investigation of treadmill and overground running: implications for the measurement of oxygen cost in children with developmental coordination disorder. Gait \& posture. 2014; 40: 464-470.

9. Chia LC, Licari MK, Guelfi KJ, Reid SL. A comparison of running kinematics and kinetics in children with and without developmental coordination disorder. Gait \& posture. 2013; 38: 264-269.

10. Diamond N, Downs J, Morris S. "The problem with running"--comparing the propulsion strategy of children with developmental coordination disorder and typically developing children. Gait \& posture. 2014; 39: 547-552.

11. Scott-Roberts S, Purcell C. Understanding the Functional Mobility of Adults with Developmental Coordination Disorder (DCD) Through the International Classification of Functioning (ICF). Curr Dev Disord Rep. 2018; 5: 26-33.

12. James EG, Leveille SG, Hausdorff JM, Barton B, Cote S, Karabulut M, et al Coordination Impairments Are Associated With Falling Among Older Adults.
Experimental aging research. 2017; 43: 430-439.

13. Carollo JJ, Worster K, Pan Z, Ma J, Chang F, Valvano J. Relative phase measures of intersegmental coordination describe motor control impairments in children with cerebral palsy who exhibit stiff-knee gait. Clinical biomechanics (Bristol, Avon). 2018; 59: 40-46.

14. Shafizadeh M, Crowther R, Wheat J, Davids K. Effects of personal and task constraints on limb coordination during walking: A systematic review and meta-analysis. Clinical Biomechanics. 2019; 61: 1-10.

15. Hollands KL, Pelton TA, Tyson SF, Hollands MA, van Vliet PM. Interventions for coordination of walking following stroke: systematic review. Gait \& posture. 2012; 35: 349-359.

16. Diedrich FJ, Warren WH. Why change gaits? Dynamics of the walk-run transition. Journal of experimental psychology Human perception and performance. 1995; 21: 183-202.

17. Downes MJ, Brennan ML, Williams HC, Dean RS. Development of a critical appraisal tool to assess the quality of cross-sectional studies (AXIS). England: BMJ Publishing Group LTD. 2016: e011458.

18. Boos CJ, De Villiers N, Dyball D, McConnell A, Bennett AN. The Relationship between Military Combat and Cardiovascular Risk: A Systematic Review and Meta-Analysis. International journal of vascular medicine. 2019; 2019: 9849465.

19. Eggleston JD, Landers MR, Bates BT, Nagelhout E, Dufek JS. Weighted Walking Influences Lower Extremity Coordination in Children on the Autism Spectrum. Perceptual and motor skills. 2018; 125: 1103-1122.

20. Meyns P, Desloovere K, Molenaers G, Swinnen SP, Duysens J. Interlimb coordination during forward and backward walking in primary school-aged children. PloS one. 2013; 8: e62747.

21. Hallemans A, Aerts P. Effects of visual deprivation on intra-limb coordination during walking in children and adults. Experimental brain research. 2009; 198: 95-106.

22. Bleyenheuft $\mathrm{C}$, Detrembleur C. Kinematic covariation in pediatric, adult and elderly subjects: Is gait control influenced by age? Clinical Biomechanics. 2012; 27: 568-572.

23. Whitall J. A developmental study of the interlimb coordination in running and galloping. Journal of motor behavior. 1989; 21: 409-428.

24. Borghese NA, Bianchi L, Lacquaniti F. Kinematic determinants of human locomotion. The Journal of physiology. 1996; 494: 863-879.

25. Miller RH, Chang R, Baird JL, Van Emmerik RE, Hamill J. Variability in kinematic coupling assessed by vector coding and continuous relative phase. Journal of biomechanics. 2010; 43: 2554-2560.

26. Burgess-Limerick R, Abernethy $B$, Neal RJ. Relative phase quantifies interjoint coordination. Journal of biomechanics. 1993; 26: 91-94.

27. Lamb PF, Stöckl M. On the use of continuous relative phase: Review of current approaches and outline for a new standard. Clinical biomechanics (Bristol, Avon). 2014; 29: 484-493.

28. Plotnik M, Giladi N, Hausdorff JM. A new measure for quantifying the bilateral coordination of human gait: effects of aging and Parkinson's disease. Experimental brain research. 2007; 181: 561-570.

29. Kribus-Shmiel L, Zeilig G, Sokolovski B, Plotnik M. How many strides are required for a reliable estimation of temporal gait parameters? Implementation of a new algorithm on the phase coordination index. PloS one. 2018; 13: e0192049.

30. Cheron G, Bouillot E, Dan B, Bengoetxea A, Draye JP, Lacquaniti F. Development of a kinematic coordination pattern in toddler locomotion: planar covariation. Experimental brain research. 2001; 137: 455-466.

31. Barliya A, Omlor L, Giese MA, Flash T. An analytical formulation of the law of intersegmental coordination during human locomotion. Experimental brain research. 2009; 193: 371-385.

32. Hallemans A, Beccu S, Van Loock K, Ortibus E, Truijen S, Aerts P. Visual deprivation leads to gait adaptations that are age- and context-specific: I. 
Step-time parameters. Gait \& posture. 2009; 30: 55-59.

33. Cheron G, Bengoetxea A, Bouillot E, Lacquaniti F, Dan B. Early emergence of temporal co-ordination of lower limb segments elevation angles in human locomotion. Neuroscience letters. 2001; 308: 123-127.

34. Stephenson J, Carter M. The use of weighted vests with children with autism spectrum disorders and other disabilities. Journal of autism and developmental disorders. 2009; 39: 105-114.

35. McGinley JL, Baker R, Wolfe R, Morris ME. The reliability of three-dimensiona kinematic gait measurements: a systematic review. Gait \& posture. 2009; 29 $360-369$.
36. Novacheck TF. The biomechanics of running. Gait \& posture. 1998; 7: 77-95

37. Yam TTT, Fong SSM. Leg muscle activation patterns during walking and leg lean mass are different in children with and without developmental coordination disorder. Research in developmental disabilities. 2018; 73: 8795. 